\title{
La aparición de las "Grow Shop \& Smartshop" y los nuevos cambios en el consumo de drogas: un estudio en sus usuarios
}

\author{
José Mendiola Alonso, Elisardo Becoña Iglesias
}

Universidad de Santiago de Compostela

Enviar correspondencia a:

Elisardo Becoña. Universidad de Santiago de Compostela. Facultad de Psicología. Departamento de Psicología Clínica y Psicobiología. Campus Universitario Sur. 15782 Santiago de Compostela. E-mail: pcelisar@usc.es.

\section{RESUMEN}

En los últimos años en España se han abierto un gran número de tiendas bajo la denominación de Grow Shop \& Smartshop en las que es posible comprar plantas, semillas, ropa, cosméticos, objetos y utensilios para el cultivo de distintas drogas. Para conocer las características de sus usuarios se hizo un estudio en dos tiendas de Santiago de Composela con 100 clientes entrevistados al azar. Se analizaron sus consumos de drogas, la compra de productos en las tiendas, junto a la organización de su vida recreativa, percepción del riesgo de las distintas drogas, obtención y tráfico con las mismas. Los resultados muestran que la mitad de ellos son consumidores habituales de cannabis, que la edad predominante de sus usuarios es de 18 a 25 años, que en general tienen una baja percepción del riesgo, un mayor nivel de vida recreativa y de conductas relacionadas con el consumo de drogas. A la luz de los resultados se discute el papel de estas tiendas en el consumo de drogas y de sus usuarios.

Palabras clave: Grow Shop, Smartshop, drogas, cannabis, consumo, tienda.

\section{SUMMARY}

In the last years in Spain a great number of shops has opened under the denomination of Grow Shop \& Smartshop. In this shops is possible to buy plants, seeds, clothes, cosmetics, objects and utensils for the cultivation of different drugs. To know the characteristics of their users a study was made in two stores of Santiago of Composela (Spain) with 100 clients interviewed at random. The drug consumptions, purchase of products in the stores, organization of their recreational life, perception of risk of the different drugs, and obtaining and traffic with the same ones were analyzed. The results show that half of them are habitual consumers of cannabis, that the predominant age of their users is from 18 to 25 years, that have a low perception of the risk in general, a bigger level of recreational life, and behaviors related with the consumption of drugs. Based on this results we discuss the role of these shops in the consumption of drugs and of the buyers.

Key words: Grow Shop, Smartshop, drugs, cannabis, consumption, shop.

\section{INTRODUCCIÓN}

$\mathbf{N}$ o cabe ninguna duda de que el estudio del consumo y de la adicción a las drogas es un campo complejo, dinámico y a veces hasta contradictorio. En el momento actual se abre una nueva, o posible nueva etapa, de evolución incierta, ante el surgimiento y la rápida eclosión de un nuevo tipo de tienda con la denominación de "Smart \& Grow Shop" contabilizándose en España 169 en el momento del estudio (1). Se trata de pequeñas tiendas que, en la mayoría de los casos, actúan como distribuidoras de productos de grandes franquicias (L'interior, Sinsemilla, Doctor cogoIlo). Tienen el denominador común de que sus actividades giran en torno a lo que se ha denominado como "Cultura Pro-Cannabis" (2). De hecho, en un inicio las tiendas existentes se dedicaban exclusivamente a la venta de productos relacionados con el cultivo de marihuana, y se daban a conocer con el nombre de Grow Shop. Actualmente, la mayoría de estas tiendas han incorporado a su oferta distintas sustancias psicoactivas que todavía no han sido prohibidas. Aquellas tiendas que incorporan esta nueva gama de sustancias conocidas como "sustancias naturales" se denominan como Smart \& Grow Shop. A través de estos comercios el consumidor tiene la posibilidad de transformar sus hábitos de adquisición y, además, ampliar el número de sustancias entre las que escoger, algunas de las cuales son de potencia considerable.

Este tipo de comercios ha encontrado su principal vehículo de promoción en la revista Cáñamo que se autodenomina como la "revista de la cultura del cannabis", revista de publicación mensual y que tiene una 
tirada de 35.000 ejemplares (3). Constantemente se presentan artículos sobre el cultivo de marihuana y estos son de gran utilidad para todo aquel que se quiera iniciar tanto en el cultivo como en su consumo. El cultivo casero de Cannabis está teniendo un auge considerable, el número de personas que optan por esta vía de abastecimiento ha crecido notablemente en los últimos años. Asimismo el consumo de Cannabis y deribados también ha aumentado notablemente como a su vez se ve reflejado en los niveles de consumo entre los jóvenes $(4,5)$.

Desde estas tiendas se facilita todo el material necesario para tener un cultivo casero. Un aspecto a destacar es la implicación en el cultivo, el conocimiento de los ciclos biológicos y la administración controlada de una cantidad notoria de droga que ha de durar por lo menos unos cuantos meses. Esto es bien distinto al sistema de adquisición habitual en el que cuando se acaba, se compra más (sin ningún problema). Habrá que determinar en futuras investigaciones si aquellos que se han pasado al autocultivo fuman más o menos que antes.

Uno de los objetivos del presente estudio es describir este tipo de locales, dada la carencia de información científica sobre los mismos, con la idea de obtener una imagen aproximada de cómo son y cómo funcionan, cómo estructuran su espacio, quién esta dando la cara al publico y cuáles son los productos que allí se pueden adquirir. El segundo objetivo es analizar los clientes de estos establecimientos, centrándonos especialmente en sus consumos de sustancias y en como éstas son incorporadas a su vida recreativa, tanto nocturna como diurna. Además veremos como utilizan la información disponible. Se trata de determinar en que medida estas tiendas pueden suponer un riesgo o un avance para aquellos que las visitan.

Siendo conscientes de que estas tiendas no ejercen el mismo atractivo para todos los jóvenes, nuestras hipótesis de partida son que esperamos encontrar como clientes de las mismas personas con niveles de consumo elevado (de distintas sustancias), un gran número de personas que trafican o que hayan traficado a pequeña escala, y un nivel de desacuerdo preocupante con las informaciones y consejos ofrecidos por las autoridades sobre las drogas. Este desacuerdo será proporcional a su nivel de consumo.

\section{MÉTODO}

\section{Participantes}

El estudio se realizó en dos tiendas Grow Shop \& Smartshop de la ciudad de Santiago de Compostela que se han abierto recientemente. El número de personas participantes en este estudio ha sido de 100. Estos fueron extraídos al azar de aquellos que visita- ron una de las dos tiendas, comprasen luego o no los productos allí expuestos. De todas las personas que visitaron la tienda hubo un número muy pequeño de sujetos que se negaron a contestar el cuestionario, ocho en total.

\section{Instrumento de evaluación}

Para recoger la información se ha empleado un cuestionario, adaptado del de IREFREA para evaluar la presencia de drogas en la vida recreativa nocturna de 5 ciudades europeas (6). Se han suprimido algunas preguntas y se han modificado otras para adaptarlas al objetivo de este estudio, especialmente por la necesidad de primar la brevedad de aplicación. En este cuestionario se recogen las características demográficas de la muestra y se plantean preguntas relativas al consumo de sustancias, a su disponibilidad y la percepción del riesgo de estos consumos. También se indaga en las fuentes de información de estos sujetos y en como manejan la información que las autoridades les facilitan.

\section{Procedimiento}

Se contactó con los responsables de las tiendas y se les pidió permiso para realizar la recogida de datos en el interior de ellas. No pusieron ningún obstáculo para la realización del estudio. Los cuestionarios se pasaron de manera ininterrumpida a lo largo de toda la jornada comercial.

El nivel de aceptación para participar en el estudio fue muy grande. De los sujetos perdidos hubo tres grupos. Uno, constituido por dos personas, que se negaron a contestar. Otro formado por un grupo de curiosos y curiosas de unos 16 - 22 años con ropa deportiva y bolsa de deportes que parecían estar tutelados por una persona mayor que nos advirtió de que no querían hacer el cuestionario; $y$, finalmente, un grupo de 3 personas que se fueron por estar todo el espacio de la tienda colapsado por un gran número de personas.

Si bien el cuestionario estaba pensado para que se cubriese en 5 minutos, algunos sujetos tardaban más de un cuarto de hora y se pensaban mucho las respuestas.

Los datos fueron recogidos entre el 1 y el 18 de junio del 2001.

\section{RESULTADOS}

\section{Descripción de las tiendas}

\section{Ubicación}

Las dos tiendas en donde se ha realizado el estudio están situadas en lugares con cierta densidad de 
trafico peatonal, una de ellas en las galerías de la zona nueva de la ciudad y la otra en el casco viejo. Nuestro conocimiento de otras tiendas en Galicia también muestra que éstas se han situado en lugares que sean accesibles y tengan un mínimo de movimiento. Lo cierto es que en los últimos dos años estos comercios han tenido un auge espectacular, abriéndose gran cantidad de ellas a lo largo de todo el territorio nacional y de otros países de la comunidad europea.

\section{Estructuración del espacio y disposición de los stands}

Sería extraño encontrarnos de repente con un comercio en el que se nos vendiese todo tipo de drogas. Sería un shock. Ante ciertas sustancias no se encontraría la misma reacción que se tiene al pasar por un estanco o por la sección de bebidas de un supermercado. Es por esto que las sustancias que allí se venden se muestran de una manera discreta y mezcladas con gran variedad de productos, algunos relacionados con el consumo y otros no. Además hay que tener en cuenta que las sustancias en sí ocupan muy poco por lo que será importante rellenar espacios con productos que puedan ser de interés a los clientes. Así, en una de las tiendas nos encontramos con que la actividad comercial esta compartida por los propietarios del comercio y por una supuesta meiga (bruja) que además de realizar las actividades propias de su oficio se dedica a hacer tatuajes y piercings, muy de moda últimamente, siendo notorio el hecho de que ya han perdido la marginalidad que les caracterizaba.

El más espacioso de los establecimientos cuenta con dos sofás y mesita en donde se puede consultar la literatura propia de la tienda (casi toda ella bajada de internet). La gente charla y pasa el rato. Este espacio fue aprovechado para la cumplimentación de los cuestionarios en esta tienda.

\section{Entrevista y trato con los dependientes}

La intención de colaboración y transparencia por parte de los encargados de las tiendas fue un tanto sorprendente, aunque esto puede variar en función del tipo de entrada que se realice. Hay una concienciación importante acerca de sus funciones como asesores a la hora de proporcionar determinadas sustancias. La impresión recibida es que estos jóvenes emprendedores no ven que el nivel de ventas sea el deseado. Lo que se presentó como un buen negocio no lo es tanto, al menos en Santiago de Compostela y durante sus meses iniciales. Además, es palpable el ambiente de incertidumbre que rodea estos comercios y sus actividades.

\section{Enumeración de los productos}

\section{No psicoactivos}

Literatura. Aparte de los numerosos documentos de consulta que los clientes pueden leer allí, cuentan con un stand en el que hay varios libros a la venta.
Como es sabido los encargados de informar acerca de este fenómeno (consumo de drogas) presentan opiniones y escritos muy polarizados.

Ropa. Según las posibilidades espaciales y económicas del comercio, podemos encontrar distintas prendas de vestir, como camisetas o pantalones. Muchas tiendas tienen camisetas con su propio logotipo y la hoja de marihuana es un clásico que siempre vende.

Parafernalia. Balanzas, inciensos, pipas, tijeras, librillos (de papel), cartones con medida especial para la confección de filtros..., y todo lo que pueda acompañar a una sesión de consumo de cannabis. También hay útiles para el consumo de cocaína (carterasespejito).

Kit de cultivo. El plato fuerte de estas tiendas es en teoría, la venta de los distintos elementos necesarios para el montaje de un cultivo propio, para lo cual es necesario un par de metros cuadrados y una inversión de unos 200 Euros para los kits mas elementales, que estarían compuestos por: bolsa de semillas con pedigree, saco de tierra, lámpara especial, ventilador (no es imprescindible), abonos y fertilizantes varios, esto es para el cultivo de la marihuana.

En algunas tiendas se pueden adquirir los elementos necesarios para un cultivo casero de hongos alucinógenos (Psilocybe cubensis). Este es más rápido y complejo y tiene que ser realizado en condiciones de máxima higiene. Se pueden adquirir jeringuillas con una disolución que contenga las esporas, y en algunas ocasiones bandejas con el micelio ya extendido. También se venden cactus jóvenes, Peyote (Lophophora williamsii) y San Pedro (Trichocereus pachanoi) que tendrán que ser atendidos bastantes años antes de poder ser consumidos.

Cosmética. Jabones, cremas, champús y aceites supuestamente fabricados de cáñamo; también gena tradicional.

Nutritivas. Piruletas, caramelos y chocolatinas.

\section{Sustancias psicoactivas}

En estos comercios se pueden adquirir lo que Miller define como droga; "sustancias farmacológicamente activas que tienen efectos químicos sobre el cerebro" (7). Si bien hay que apuntar que muchos consumidores consideran "nula" o muy suave la actividad farmacológica de lo que se les ha vendido como droga, del otro lado están sustancias como "la Salvia" que algunos consideran demoledora.

Tratar una por una a las sustancias sería excesivo y no es el objeto del presente estudio, por lo que se enumeraran aquellas sustancias ofertadas a través de distintos anuncios, como frecuentemente puede verse en la revista Cáñamo: Piper methysticum, salvia divinorum, ephedra, rosa lisergica, jurema, pedicularis atollens, kanna, yohimbe, kavakava, lactua, nuez de 
cola, damiana, guarana sinicuiche, harmala, mimosa hostilis, nepetea cataria, galangal (8).

Los denominados como "éxtasis herbales" se supone que son preparados a base de los principios activos de algunas plantas, las combinaciones son varias y los efectos prometidos también, yendo desde: "risa y distorsión visual; colores con más briIlo", a "la balanza perfecta entre energía y euforia"(9). La mayoría de ellas están preparadas a base de: Ephedra, yohimbe, guaraná y otros suplementos energéticos (10). En general, tienen muy poca aceptación por ser consideradas demasiado suaves frente a los "éxtasis callejeros". Hay una gran cantidad de firmas que se dedican a la comercialización.

\section{Características sociodemográficas de la muestra:}

En la tabla 1 se indican las características sociodemográficas de las personas a las que se les pasó el cuestionario, que son las personas que entraron en las tiendas para comprar o ver que había en ella.

\section{Tabla 1. Características sociodemográficas de} la muestra

\begin{tabular}{llr}
\hline Variable & Grupo & Porcentaje \% \\
\hline SEXO & Hombres & 68 \\
EDADES & Mujeres & 32 \\
& Menores de 18 & 11 \\
& Entre 18 y 22 & 56 \\
ESTADO CIVIL & Mayores de 22 & 33 \\
& Soltero(a) & 97 \\
& Casado & 1 \\
ESTUDIOS & Separada & 1 \\
& Universitarios & 46 \\
& BUP COU & 29 \\
& ESO - EGB & 10 \\
& FP-Técnicos & 11 \\
& Oposiciones & 1 \\
CONVIVENCIA & Militares & 1 \\
& Familia & 49 \\
& Amigos & 24 \\
& Solo & 16 \\
& Pareja & 5 \\
& Residencia & 2 \\
& Con hijos & 1 \\
STATUS & No contesta & 3 \\
& Alto & 1 \\
& Medio-alto & 14 \\
& Medio & 61 \\
& Medio-Bajo & 18 \\
& Bajo & 6 \\
\hline
\end{tabular}

\section{Características recreativas}

En estos jóvenes es importante conocer como pasan el tiempo libre, dándole una especial relevancia a la conducta entendida como "salir de marcha" sobre la que se profundizó algo más. Se pretendía tener una idea aproximada con respecto a otras actividades que también son practicadas por la mayoría de los jóvenes y ver si había diferencia en la satisfacción producida por esas actividades en el caso de que las realizasen o no bajo el efecto de algunas sustancias. Se esperaba encontrar por ejemplo que la actividad salir de marcha es más satisfactoria cuando se realiza "colocado".

La evaluación de las conductas que se realizan sin la mediación de alguna sustancia se indican en la tabla 2 , donde destacan principalmente estar con los amigos y sexo, que son de lo más satisfactorio, seguido de excursiones cortas, viajes largos, salir de marcha y lectura, seguido a cierta distancia de las demás.

Las mismas actividades han sido evaluadas en el caso de que fueran realizada bajo el efecto de alguna droga (tabla 3). Los resultados aquí indican el mayor nivel de satisfacción en estar con los amigos, salir de marcha y excursiones cortas, seguido por sexo, viajes largos y cine/televisión, junto a otras con menor peso. Se introdujo el item "me gustaría practicar para tener una idea aproximada de que actividades podrían resultar alternativas a salir de marcha" de cara a realizar programas preventivos o de reducción de daños. Con diferencia son internet y los deporte de riesgo las actividades más deseadas.

\section{Salir de marcha}

Se les preguntó con qué frecuencia salían de marcha en un mes normal (tabla 4). Para evaluar la frecuencia con la que se sale de noche se ha utilizado como posible respuesta la afirmación "siempre que puedo". La información obtenida es cuantitativamente escasa, pues no se nos aclara si salen mucho o salen poco; pero se trata de un dato de alto valor cualitativo, pues esta refiriéndose hacia las intenciones y posibilidades del encuestado. Resultó que un $49 \%$ de la muestra sale "siempre que puede", y tan solo el 16\% de la muestra sale con una frecuencia igual o inferior a 2 veces por mes.

Con respecto al número de horas que duran estas salidas, encontramos que la mayoría (64\%) sale entre 6 y 8 horas. Vemos que un $9 \%$ estaría por debajo de las seis horas y un $25 \%$ por encima de las 8 horas.

El dinero gastado de cada salida se sitúa entre las 1000 y 3000 pts. (6 y 18 euros) para un 69\% de la muestra, encontrándose tan solo 3 individuos que gasten menos de 1000 pts. (6 euros). Con respecto a la procedencia del dinero gastado durante el fin de semana se les pregunto de tal manera que podían 
Tabla 2: Evaluación de las actividades cuando se realizan libres de drogas (en porcentajes).

\begin{tabular}{lccccc}
\hline & $\begin{array}{c}\text { NO } \\
\text { PRACTICO }\end{array}$ & $\begin{array}{c}\text { ME GUSTARÍA } \\
\text { PRACTICAR }\end{array}$ & $\begin{array}{c}\text { POCO } \\
\text { SATISFACTORIO }\end{array}$ & $\begin{array}{c}\text { SATISFACCIÓN } \\
\text { NORMAL }\end{array}$ & $\begin{array}{c}\text { MUY } \\
\text { SATISFACTORIO }\end{array}$ \\
\hline ESTAR CON AMIGOS & 0 & 0 & 4 & 24 & 65 \\
SEXO & 3 & 6 & 1 & 12 & 69 \\
SALIR DE MARCHA & 2 & 1 & 9 & 21 & 41 \\
TV, CINE... & 1 & 1 & 1 & 45 & 34 \\
INTERNET & 20 & 3 & 12 & 36 & 19 \\
VIDEO JUEGOS & 29 & 1 & 8 & 33 & 13 \\
JUEGOS DE AZAR & 56 & 1 & 13 & 11 & 4 \\
EXC. CORTAS & 5 & 4 & 8 & 14 & 54 \\
VIAJES LARGOS & 8 & 7 & 4 & 24 & 46 \\
DEP. CONVENCIONALES & 21 & 4 & 5 & 30 & 31 \\
DEP. DE RIESGO & 29 & 30 & 1 & 7 & 21 \\
VOLUNTARIADO SOCIAL & 57 & 7 & 6 & 12 & 7 \\
LECTURA & 10 & 3 & 6 & 29 & 40 \\
OTRAS & 2 & 0 & 2 & 3 & 4 \\
\end{tabular}

Tabla 3. Evaluación de las actividades cuando son realizadas bajo el efecto de alguna sustancia (en porcentajes).

\begin{tabular}{lccccc}
\hline & $\begin{array}{c}\text { NO } \\
\text { PRACTICO }\end{array}$ & $\begin{array}{c}\text { ME GUSTARÍA } \\
\text { PRACTICAR }\end{array}$ & $\begin{array}{c}\text { POCO } \\
\text { SATISFACTORIO }\end{array}$ & $\begin{array}{c}\text { SATISFACCIÓN } \\
\text { NORMAL }\end{array}$ & $\begin{array}{c}\text { MUY } \\
\text { SATISFACTORIO }\end{array}$ \\
\hline ESTAR CON AMIGOS & 0 & 0 & 0 & 17 & 68 \\
SEXO & 4 & 5 & 3 & 16 & 41 \\
SALIR DE MARCHA & 1 & 0 & 0 & 12 & 69 \\
TV, CINE... & 1 & 5 & 4 & 18 & 33 \\
INTERNET & 11 & 12 & 6 & 9 & 8 \\
VIDEO JUEGOS & 7 & 0 & 4 & 16 & 17 \\
JUEGOS DE AZAR & 17 & 1 & 4 & 2 & 2 \\
EXC. CORTAS & 2 & 3 & 1 & 7 & 62 \\
VIAJES LARGOS & 5 & 6 & 3 & 3 & 39 \\
DEP. CONVENCIONALES & 12 & 4 & 9 & 6 & 3 \\
DEP. DE RIESGO & 15 & 9 & 3 & 2 & 3 \\
VOLUNTARIADO SOCIAL & 18 & 4 & 3 & 1 & 1 \\
LECTURA & 6 & 2 & 7 & 16 & 15 \\
OTRAS & & & & & \\
\end{tabular}

contestar dos posibilidades simultáneamente, se tuvo en cuenta el hecho de que las fuentes de ingreso en esta franja de edad pueden ser variadas.

Se introdujo la posibilidad de contestar "algún trapicheo" para determinar el número de pequeños traficantes en activo. Resultó que un total de 15 sujetos están actuando como camellos, a pesar del riesgo que conlleva. Tan solo uno de ellos lo marca como su única fuente de ingresos para el fin de semana.

Es la familia, para la mayoría, la que se encarga de sufragar las salidas nocturnas del fin de semana.

\section{Policonsumo}

A la pregunta de si tomaban más de una sustancia en la misma noche, un $\mathbf{7 4} \%$ contestó afirmativamente. En otra pregunta se les pidió que escribiesen las combinaciones que solían hacer, dejándoles tres renglones para que pudiesen escribir diferentes combinaciones. Los resultados de las combinaciones más relevantes practicadas por estos 74 sujetos se muestran en la tabla 5. Se puede ver que las sustancias combinadas con más frecuencia son el alcohol y el cannabis (50\%), a las que se les suele añadir algún 
Tabla 4. Cuestiones relativas a la conducta de salir de marcha

\begin{tabular}{lr}
\hline Cuestiones & $\%$ \\
\hline Frecuencia con la que sales & \\
$\quad$ Siempre que puedo & 49 \\
3 o 4 veces al mes & 19 \\
Más de 4 veces al mes & 16 \\
1 o 2 veces al mes & 12 \\
Menos de1 o 2 veces al mes & 4 \\
\hline \multicolumn{2}{l}{ Cuantas horas sales } \\
$\quad$ Entre 6 y 8 horas & \\
Más de 8 horas & 64 \\
Menos de 6 horas & 25 \\
\hline & 9 \\
Dinero gastado & 3 \\
$\quad$ Menos de 1000 pts & 69 \\
Entre 1000 y 3000 pts & 24 \\
Entre 3000 y 7000 pts & 4 \\
Más de 7000 pts
\end{tabular}

Procedencia del dinero gastado

durante el fin de semana

$\begin{array}{lr}\text { Familia } & 45 \\ \text { Trabajo temporal } & 15 \\ \text { Trapicheo } & 15 \\ \text { Trabajo fijo } & 14 \\ \text { Becas } & 9 \\ \text { Otros } & 2\end{array}$

Puede ser peligroso como única forma de diversión

No peligroso

Poco peligroso

Peligro moderado

Muy peligroso

estimulante poderoso como la cocaína o pastillas para poder aguantar más tiempo (19\% y 13\%). Los alucinógenos como los tripis o los hongos son los menos mezclados (43 sujetos dicen haberlos probado y solo 3 los mezclan) separándose a menudo del contexto recreativo nocturno para ser utilizados en momentos más libres de presiones e intromisiones. La heroína no aparece.

\section{Conocimiento de las sustancias y consumo}

Para ver el grado de conocimiento y de consumo que se tenia sobre las sustancias vendidas en estas tiendas se incluyeron en el cuestionario algunas preguntas relativas a las mismas. En relación a si habían

\section{Tabla 5. Policonsumo}

\begin{tabular}{lc}
\hline Combinación de sustancias & No sujetos $^{\circ}$ \\
\hline Alcohol + cannabis & 50 \\
Alcohol + cannabis + cocaína & 19 \\
Alcohol + cannabis + pastillas & 13 \\
Éxtasis herbal + pastillas + cannabis & 1 \\
Alcohol + cocaína & 7 \\
Cocaína + (sin alcohol) & 6 \\
Alcohol + pastilla & 12 \\
Pastilla + (sin alcohol) & 3 \\
Hongos, tripis + (.......) & 6 \\
Otros &
\end{tabular}

oído hablar de nuevas drogas y qué nombres recibían, se encontró que tanto las sustancias distribuidas por estos comercios como las distribuidas en el mercado negro son consideradas novedosas. Aparecen 52 sujetos que no contestan, o que no han oído hablar de nuevas drogas, 23 hablan de drogas naturales, (que así se denomina a esta nueva saga de sustancias que se ofrecen en estas tiendas, comercialmente se conocen como productos smart), 10 marcan la ketamina y 8 el éxtasis liquido.

Posteriormente, para evaluar la percepción de riesgo que se tiene de estas sustancias, se les pregunto si creían si las sustancias que se podían adquirir en el comercio estaban exentas de peligros (tabla 6). Hay un total de 27 sujetos que consideran que estas sustancias están exentas de peligro y, en general, se aprecia frente a ellas una actitud de prudencia y de respeto.

Para profundizar en la cuestión de la percepción del riesgo de estas sustancias se les pregunto sobre cuales creían que eran más peligrosas. En esta pregunta a pesar de que se les indicó que se refería a las sustancias que se podían adquirir en el comercio la

\section{Tabla 6. Drogas naturales}

¿Estas sustancias están exentas de peligros?

$\mathrm{Si}$, exentas de peligro 27

No, son peligrosas 20

Depende (dosis, consumidor) 16

No lo sé, no contesta 17

¿Cuáles crees que son más peligrosas?

Alucinógenos fuertes 6

Salvia 8

Cactus 3

Hongos 5 
mayoría de las respuestas se orientaron hacia las drogas ilegales más conocidas como la heroína, cocaína o pastillas. En total solo hubo 22 personas que hicieron alusión a las drogas del comercio. De las sustancias vendidas en la tienda sólo los alucinógenos potentes están considerados como peligrosos, y la única de estas sustancias que puede ser adquirida lista para su consumo es la "Salvia" ya que en el caso de los hongos y los cactus lo que se vende no puede ser consumido directamente, sino que hay que cultivarlo.

\section{Sustancias consumidas: Frecuencia, disponibilidad y percepción del riesgo}

Se va a ver con que frecuencia son consumidas las distintas drogas que ofrece el mercado, sin entrar en las características farmacológicas de unas o de otras. La percepción del riesgo es una variable de gran relevancia para explicar el consumo o no de sustancias. Las personas toman decisiones en función de las consecuencias positivas que van a obtener y evitan las consecuencias negativas. Si perciben que algo les va acarrear dichas consecuencias negativas no lo harán. Por ello, la concepción que se tiene sobre las distintas drogas, que depende tanto del uso, como de las creencias y de la propia construcción social sobre la sustancia, influye sobre su consumo (11).

Otro factor que se relaciona claramente con el consumo de drogas es que éstas estén disponibles para los posibles consumidores; si no están disponibles, el consumo es difícil o puede llegar a hacerse imposible. Se ha introducido la posibilidad de responder "nunca lo he intentado" para marcar la diferencia en aquellas drogas que no se consumen por que no interesa su consumo, al margen de que sean fácilmente localizables, como es el caso de la heroína. En la tabla 7 se muestra la frecuencia, disponibilidad y percepción del riesgo para las distintas sutancias.

Tabaco. Se trata de la sustancia más consumida diariamente. La percepción de riesgo de fumar cigarrillos al día es más alta que la de fumar hachís habitualmente, lo que resulta indicativo del alcance e impacto de las actuales campañas antitabaco.

Alcohol. Es la droga que más se consume de forma semanal. Su uso es considerado más o menos peligroso dependiendo de las dosis y frecuencia (tabla 8). En el caso de beber todos los fines de semana, algo que el $70 \%$ de ellos realiza, resulta que tan solo un $22 \%$ lo considera no peligroso. Para aquellos consumos diarios de no más de dos bebidas/ día (consumo realizado por una parte considerable de la población) encontramos que el $71 \%$ lo considera al menos un poco peligroso.

Con respecto al consumo de 4 bebidas alcohólicas en una ocasión, resulta ser que es la conducta con respecto al alcohol que es considerada como menos peligrosa, un $53 \%$ cree que no es peligroso su ingesta.

Tabla 7. Frecuencias de consumo, disponibilidad y percepción del riesgo de las distintas sustancias

\begin{tabular}{|c|c|c|c|c|c|c|c|c|c|}
\hline & Tabaco & Alcohol & \multicolumn{2}{|c|}{ Cannabis } & Pastillas & Cocaína & Heroína & \multicolumn{2}{|c|}{ Alucinógenos } \\
\hline \multicolumn{10}{|l|}{ Frecuencia de consumo } \\
\hline - Diario & 69 & 13 & \multicolumn{2}{|c|}{53} & 0 & 0 & 0 & \multicolumn{2}{|c|}{1} \\
\hline - Semanal & 6 & 70 & \multicolumn{2}{|c|}{20} & 10 & 6 & 0 & \multicolumn{2}{|c|}{2} \\
\hline - Mensual & 3 & 11 & \multicolumn{2}{|c|}{12} & 23 & 29 & 0 & \multicolumn{2}{|c|}{9} \\
\hline - Anual & 3 & 2 & \multicolumn{2}{|c|}{3} & 18 & 0 & 4 & \multicolumn{2}{|c|}{31} \\
\hline - Nunca & 19 & 4 & \multicolumn{2}{|c|}{12} & 49 & 65 & 96 & \multicolumn{2}{|c|}{57} \\
\hline Disponibilidad & & & $(1)^{*}$ & $(2)^{*}$ & & & & Tripis & hongos \\
\hline - Muy fácil & 93 & 94 & 22 & 59 & 20 & 18 & 10 & 13 & 13 \\
\hline - Fácil & 5 & 5 & 36 & 28 & 39 & 35 & 9 & 28 & 9 \\
\hline - Difícil & 0 & 0 & 26 & 1 & 3 & 12 & 7 & 17 & 28 \\
\hline - Muy difícil & 0 & 0 & 3 & 0 & 1 & 1 & 1 & 1 & 6 \\
\hline - Nunca lo he intentado & 2 & 1 & 13 & 12 & 37 & 34 & 73 & 41 & 44 \\
\hline Riesgo & Diario & Diario & & rio & Fines de & Fines de & Fines de & & vez \\
\hline de consumo & & & & & semana & semana & semana & & mes \\
\hline - No peligroso & 10 & 29 & & & 1 & 1 & 0 & & 11 \\
\hline - Un poco peligroso & 22 & 28 & & & 7 & 7 & 0 & & 28 \\
\hline - Peligro moderado & 36 & 29 & & & 32 & 32 & 5 & & 41 \\
\hline - Muy peligroso & 32 & 14 & & & 60 & 60 & 95 & & 20 \\
\hline
\end{tabular}




\begin{tabular}{|l|ccc|}
\hline \multicolumn{4}{|c|}{ Tabla 8. Percepción del riesgo del consumo de alcohol } \\
\hline \multicolumn{4}{|c|}{ Riesgo de beber } \\
\hline & $\begin{array}{c}\text { Diariamente } \\
\text { (2 bebidas alcohólicas) }\end{array}$ & Los fines de semana & $\begin{array}{c}\text { 4 bebidas alcohólicas } \\
\text { en una ocasión }\end{array}$ \\
\hline No peligroso & 29 & 22 & 53 \\
Un poco peligroso & 28 & 31 & 26 \\
Peligro moderado & 29 & 35 & 17 \\
Muy peligroso & 14 & 12 & 4 \\
\hline
\end{tabular}

Su disponibilidad, al igual que la del tabaco, un $94 \%$ cree que es muy fácil, un $5 \%$ que es fácil, y una persona dice nunca haberlo intentado.

Cannabis. Después del tabaco, es la droga que más se consume de forma diaria. El cannabis resulta la sustancia mayormente escogida para consumir. En total un $53 \%$ de los encuestados lo consumen diariamente.

En el apartado de la disponibilidad se ha introducido la diferenciación entre las distintas opciones de compra que puede escoger el consumidor. Con respecto al denominado como costo 1, en cuya categoría se incluyen los llamados "huevitos" y el polen fresco y de calidad cuyo precio oscila entre 700 y 1200 pts/gr (al por menor) vemos que el tema de la compra se complica un poco marcando un $22 \%$ de los encuestados que les resulta difícil su adquisición. Se ha denominado costo 2 al hachís malo y barato, donde las diferencias de precio y calidad son evidentes. Hay un $1 \%$ de la muestra que considera que es difícil de conseguir. Su precio oscila entre 5000 y 7000 pts. los 25gr.

En el caso de la marihuana (tabla 9), vemos que de todas las sustancias del mercado negro es la más difícil de conseguir. Un $59 \%$ de sujetos creen que es difícil, o muy difícil de conseguir. Esto es así porque es muy extraño encontrar a cultivadores con superávit que se permitan el vender.

\section{Tabla 9. Disponibilidad de marihuana}

\begin{tabular}{lc}
\hline & Marihuana \\
\hline Muy Fácil & 8 \\
Fácil & 19 \\
Difícil & 45 \\
Muy difícil & 14 \\
Nunca lo he intentado & 19
\end{tabular}

En el caso de la percepción del riesgo (tabla 10) de los consumos de cannabis nos encontramos que estos se hayan claramente menospreciados. Conside- ran que el consumo diario de cannabis es menos nocivo que el consumo de tabaco (un $23 \%$ consideran que no es peligroso frente al $10 \%$ que no consideran peligroso el consumo de una cajetilla de tabaco). Esto indica en esta muestra el interés y auge que tiene el cannabis, junto a la consideración de que la misma no causa problemas, aunque esta creencia sea errónea. En el caso de que su consumo sea esporádico se trata de la sustancia más segura para nuestra muestra (un $80 \%$ considera que su consumo esporádico no es peligroso). La falta de estudios y sobre todo el desconocimiento - interesado o no - de los estudios existentes ha facilitado la extensión de diversas ideas erróneas sobre el cannabis en la población general (12).

\section{Tabla 10. Percepción de riesgo de fumar cannabis. Diariamente (varios al día) vs. esporádicamente}

\begin{tabular}{lcc}
\hline & Diariamente & Esporádicamente \\
\hline No peligroso & 23 & 80 \\
Un poco peligroso & 47 & 16 \\
Peligro moderado & 27 & 3 \\
Muy peligroso & 3 & 1
\end{tabular}

Pastillas. Encontramos que casi la mitad de la muestra nunca ha consumido pastillas. Y que un 10\% de la misma las consume de forma semanal. Su disponibilidad en el mercado es alta ya que 59 personas consideran que su compra es fácil o muy fácil. Los que no compran es porque no quieren, solo 4 personas consideran su compra como difícil o muy difícil.

La percepción del peligro asociado a su consumo también se ha evaluado en función de las frecuencias del mismo encontrándose que hay un cierto temor a los consumos repetidos de esta sustancia. Su consumo repetido de forma semanal es considerado como muy peligroso por el $60 \%$ y como de peligro moderado por el $32 \%$; solamente una persona considera que su consumo semanal se trata de una practica no peligrosa. 
Cocaína. Se trata de una sustancia que es algo menos consumida que las pastillas. En esta muestra no encontramos consumos diarios y muy pocos consumos semanales. La percepción del riesgo es alta cuando se trata de consumos semanales (curiosamente es exactamente igual a la evaluación realizada por consumos de pastillas). Sin embargo, baja mucho cuando estos consumos son mensuales (que son los mayormente practicados, un $29 \%$ en total) (tabla 11).

\section{Tabla 11. Percepción del riesgo de consumo de cocaína. Fines de semana vs. una vez al mes}

\section{Fines de semana Una vez al mes}

No peligroso

Un poco peligroso

Peligro moderado

Muy peligroso
1

7

32

60
13

25

38

24
Heroína. Se trata de una sustancia en decadencia en la cual los datos epidemiológicos parecen indicar que actualmente menos gente se inicia en ella que hace unos años (5). Sus viejos adeptos muestran una imagen social que muy poco atrae a los nuevos consumidores de drogas. Es claramente la sustancia menos consumida en nuestra muestra. Se encontró gente que prefirió experimentar con el opio. Como cabía esperar la percepción del riesgo es la más alta (95\% consideran su consumo como muy peligroso).

Alucinógenos. De aquellos sujetos que han consumido, la mayoría lo hacen de forma anual, y son solo un $9 \%$ que realizan consumos mensuales. La percepción del riesgo es similar a la de consumir cocaína de forma mensual. La disponibilidad se evaluó de forma separada para las dos sustancias de este tipo más consumidas, los tripis y los hongos, teniendo en cuenta que diversas especies del género Psylocibe son fácilmente localizables en muchos montes de la geografía peninsular. Los resultados son similares (ver tabla 7).

\section{Las drogas favoritas}

Una de las preguntas incluidas en el cuestionario hacia referencia a cuales eran sus sustancias preferidas. Podían escoger libremente, independientemente de que su sustancia de elección fuese legal o ilegal. Tenían que responder tres sustancias por orden de preferencia. El primer puesto fue adjudicado de la siguiente manera: Cannabis y derivados: 62, Alcohol: 11, Cocaína: 9, Hongos: 5, Pastillas: 3. En segundo lugar: Cannabis y derivados: 35, alcohol: 24, hongos: 9, Cocaína: 7. Y, en tercer lugar Alcohol:15, Tabaco:10,
Pastillas: 10, Salvia: 10. Parece ser que a esta muestra lo que más consumen es cannabis, en cualquiera de sus presentaciones, siendo la marihuana la favorita y paradójicamente la más difícil de conseguir.

\section{Manejo de la información}

En el cuestionario se les realizó un par de preguntas de cara a evaluar el alcance y la efectividad de las actuales campañas preventivas, primeramente se les pregunto si conocían alguna campaña informativapreventiva, la respuesta es que si, mayoritariamente (74\%). La siguiente pregunta era si se sentían lo suficientemente informado sobre drogas, constestando: Si: $64 \%$, No: $35 \%$, N.C.: $1 \%$.

Después se les preguntó sobre cuales eran sus fuentes de información. Las respuestas fueron abiertas y hubo 33 personas que no contestaron. Otros contestaron más de una fuente informativa, siendo aquellas que hacían referencia a las experiencias propias o de sus iguales las que aparecieron con más frecuencia (40\%). Las respuestas se agruparon de la siguiente manera:

-Amigos, experiencias propias o de amigos, en la calle: 40

- Libros: 25

- Revistas:19

- Televisión y medios de comunicación: 12

- Campañas (folletos informativos): 4

- Internet: 4

- Familia: 3

- Colegio, instituto: 3

También se les preguntó de forma abierta que es lo que opinaban de las campañas preventivas gubernamentales, el tipo de respuesta era abierta y hubo 25 sujetos que no contestaron. La evaluación realizada por parte de los encuestados es esta:

- Bien evaluadas: 16. Incluiría respuestas como estas: "bastante buenas"; "me parecen muy bien y ayudan a mucha gente"; "que el anuncio está coñero"; "están muy bien porque es esencial que la gente esté informada"; "quizás deberíamos de recibir más información de ese carácter"; "están genial, pues hacen una buena labor".

- Mal evaluadas: 40. Algunos ejemplos: "Fatal, poca publicidad"; "no informan mucho"; que "informan sobre lo que quieren porque suelen exagerar para prevenir"; que "no responden a la realidad de los jóvenes"; "no llegan a la gente"; "no están suficientemente curradas"; "dan más ganas de meterte movidas"; "tratan a la gente joven como analfabetos, buscan el impacto y se alejan de la información".

- Evaluación ambigua: 19. "Regular"; "mas o menos", que "muchas veces no saben de lo que 
hablan pero una de las ultimas ha sido muy buena"; "que lo pasen bien"; "son muy radicales"; "un poco crueles"; "te hacen pensar en el momento pero enseguida se te olvidan los mensajes que quieren decir".

\section{Tráfico a pequeña escala}

Para indagar sobre el tráfico a pequeña escala se les preguntó si alguna vez habían vendido sustancias ilegales. En la tabla 12 puede verse que 49 personas declaran que alguna vez han vendido sustancias ilegales y 15 de ellos lo siguen haciendo en la actualidad. Los motivos por los que lo han hecho son por consumir gratis, ganar dinero o mejorar la posición en el grupo.

Tabla 12. Cuestiones relativas al tráfico a pequeña escala

¿Alguna vez has vendido sustancias ilegales? $\mathrm{Si}$

No

¿Por qué lo has hecho?

Consumir gratis

Ganar dinero

Mejorar posición en el grupo

Las tres anteriores o solo dos

Otros

\section{DISCUSIÓN}

Este estudio realizado en dos tiendas Grow Shop \& Smartshop muestra como se van abriendo espacio dentro de los consumidores de drogas, sobre todo entre los comprendidos entre 18 y 25 años de edad y que ya han consumido otras sustancias previamente. Como ocurre igualmente con el consumo de todas las drogas, la preferencia de estos comercios es mayor en los varones que en las mujeres, en una relación de 2 a 1 favorable a los varones. También destaca en esta muestra que, al haberse realizado en una ciudad universitaria, la mitad de los compradores son universitarios, siendo el resto fundamentalmente también estudiantes de BUP, COU, FP o ESO. Esto implica que hay una clara relación entre estar estudiando y acceder a estas tiendas, al tener más tiempo libre, y a la posibilidad de conocer y poder acceder al consumo de distintas drogas, tanto legales como ilegales.

Las tiendas suelen estar situadas en lugares accesibles, teniendo a la venta una gran cantidad de material relacionado con el consumo de drogas y también

diversas plantas para cultivar como el kit de cultivo. Están a la última y utilizan profusamente Internet para acceder a distinta información y a revistas especializadas orientadas a los consumidores de drogas o proconsumo.

El perfil de estos jóvenes sobre la diversión es semejante a la de los de su edad, ya que los dos motivos más importantes que hacen cuando no consumen drogas y que les son más satisfactorios son estar con amigos y buscar sexo, seguido de hacer excursiones cortas, viajes largos, salir de marcha, lectura y ver la televisión o ir al cine. En cambio, cuando están bajo los efectos de las drogas su mayor satisfacción la obtienen estando con los amigos, saliendo de marcha o haciendo excursiones cortas. También, como los otros jóvenes, salen de marcha cuando pueden siendo la familia la fuente fundamental de obtención de dinero para hacerlo, como para el resto de sus gastos, aunque 15 de ellos reconocen que obtienen también dinero del trapicheo, lo que muestra la relación entre acudir a estas tiendas y tener un mayor consumo e implicación con las drogas, lo cual era de esperar. En la misma línea, el consumo de distintas sutancias es importante, habiendo un gran número de ellos, el $74 \%$ que han consumido distintas sustancias, incluyendo las ilegales, lo que es un porcentaje superior al esperable en estos grupos de edad $(4,5)$.

El consumo de drogas legales en las personas que acuden a estas tiendas es claramente superior al de las personas equivalentes de su edad, si las comparamos con los datos epidemiológicos equivalentes, como la encuesta escolar del Plan Nacional sobre Drogas (5) o la de la población general (13). Así el 69\% consume tabaco a diario, un $13 \%$ alcohol a diario y un $70 \%$ semanalmente, destacando que un $53 \%$ de ellos consume cannabis diariamente. Esto muestra claramente que las personas que acuden a estas tiendas son predominantemente, al menos la mitad de ellos, consumidores habituales de cannabis y acuden a estas tiendas tanto para buscar información o productos de cultivo relacionados con la misma o con otras drogas consideradas de tipo "natural." Esto se ve reflejado en la valoración que hacen del riesgo de las distintas drogas, donde consideran más peligroso el consumo de tabaco y alcohol que el de cannabis. En cambio le dan una alta puntuación al nivel de peligrosidad de las otras drogas ilegales, como la cocaína, pastillas o heroína. Y, cuando se les pregunta por el consumo esporádico de cannabis, consideran que no tiene apenas peligrosidad. Esta cuestión merece un interés especial dado que el cannabis es una sustancia cuyo consumo está en auge y convencer a sus seguidores de que la misma puede traer problemas no va a ser tarea fácil.

Dado que el $49 \%$ de esta muestra reconoce que alguna vez ha vendido sustancias ilegales, hay una relación entre consumo y tráfico ilegal de drogas que 
puede acarrear distintos problemas a estas personas. Queda abierta la importante cuestión del por qué de tan alto porcentaje de trapicheo en esta muestra, aunque el objetivo de este estudio no era analizar esta cuestión. Aún así el dato deja bien clara la implicación que tiene el consumo tan extendido de distintas sustancias, como ocurre con el cannabis en esta muestra.

Lo cierto es que por el tipo de ventas y por los clientes que acuden a este tipo de tiendas las mismas podrían ser uno de los sitios adecuados para la puesta en práctica de planes de reducción de daños. Sería oportuno, ya que nos encontramos con sujetos que leen bastante (tabla 2 y 3), la confección de amplios materiales informativos en cuya elaboración se tuviese en cuenta las aportaciones de sujetos consumidores, pues esto parece que le otorgaría cierta credibilidad. Asimismo la exposición de dichos materiales podría realizarse en los comercios, pues de seguro se contaría con su colaboración. Se trata de aprovechar un espacio físico en el cual sabemos que se concentran un número importante de sujetos consumidores. En teoría también deberían de jugar un papel importante en lo relativo al trafico ilegal, pues se les ofrece a los clientes la posibilidad de cambiar las vías de adquisición de sustancias. Se supone que los fumadores de cannabis podrían cultivarlo en vez de comprarlo y que los amantes de la noche encontrarían en las drogas naturales una alternativa al consumo de éxtasis y cocaína.

Lo que este estudio sugiere es que el papel de estos comercios puede probablemente llevar a un incremento en el número de cultivadores de cannabis, al menos, por la posibilidad de poder conseguir directamente en los mismos las semillas y todos los útiles de cultivo para ellas. Aunque se podría decir lo mismo para otras drogas, el perfil de las personas que acuden y sus consumos, no lo permite afirmar tan claramente. Además, muchas plantas tienen un periodo largo de crecimiento para poder conseguir componentes psicoactivos y exigen muchos cuidados. Esto sería más un trabajo artesanal que industrial y la producción sería baja.

Hay el riesgo, en cambio, con las sustancias que venden con propiedades alucinógenas, las cuales están valoradas como "fuertes" por aquellos que las han consumido. Conviene recordar que el consumo de alucinógenos no crea dependencia y que la intensidad de la experiencia hace que muchos que los han probado no repitan, aunque para otros quizás en esto está justamente el interés (14). Pero ello no significa que no estén exentas de riesgos y que no sea un elemento de prueba o escalada hacia el consumo de otras drogas o de la propia dependencia (15).

Hay otro aspecto que hay que analizar en otros estudios en relación con estas tiendas, como la propia observación nos ha mostrado: en ocasiones las personas que preguntan o compran algunas de estas sus- tancias lo hacen, más que por las propiedades farmacológicas de las mismas, por el contexto cognitivo, subjetivo y trascendente en el que se enmarcan estos consumos, al desligarlos más de la experiencia lúdica para intentar convertirse en una experiencia constructiva y de comprensión de otras formas de entender "la vida" como son el chamanismo y otras doctrinas en auge $(16,17)$. En este caso serían sustancias de búsqueda personal o de la propia identidad o trascendencia.

Antes de finalizar debemos indicar que la situación ha cambiado desde junio de 2001, fecha en que se recogieron los datos. Entonces las drogas naturales se anunciaban y se ofrecían en la mayoría de las tiendas. A raíz de unas intervenciones de sanidad en diferentes tiendas se corrió la voz de que estaban requisando material. Los distribuidores dicen que se trata de una mercancía problemática y poco rentable, por lo que las tiendas aparentemente ya no cuentan con estos productos y se dedican exclusivamente al tema grow (cultivo de cannabis) y parafernalia. La revista Cañamo ya tiene una hermana, Yerba; que es la versión españolizada de la mítica High Times americana (18). En estas revistas ya no se anuncian productos smart pero, curiosamente, aparecen en ocasiones anuncios del Plan Nacional sobre Drogas (19).

En la calle se aprecia que los consumidores de cannabis cada vez tienen mas disponible la marihuana, aunque muchos prefieren seguir consumiendo hachís. Se precisa continuar haciendo estudios para ver la evolución de este tipo de tiendas y de su clientela.

En conclusión, este estudio muestra el auge de este tipo de tiendas, como los clientes en la mitad de los casos ya son consumidores habituales de marihuana, que tienen una menor percepción de riesgo sobre el efecto de las drogas, y que suelen ser personas previamente iniciadas en consumos de una gran variedad de drogas. Y que en dichas tiendas se vendían drogas, en el sentido químico o científico, o semillas que las van a permitir obtener en un tiempo. De ahí que sea necesario prestar más atención a este tipo de tiendas, sus productos, sus compradores y la relación que tienen en el consumo o contención del mismo en las drogas ilegales, especialmente en las que venden.

\section{REFERENCIAS BIBLIOGRÁFICAS}

(1) Listado de las Grow \& Smartshop sacado de la página de internet: www.carlosfresneda.com.

(2) Calafat, A.; Juan, M.; Becoña, E.; Fernandez, C.; Gil, E.; Llopis, J.J.; (2000). Estrategias y organización de la cultura pro-cannabis. Adicciones, 12, Supl. 2, 231-273.

(3) Diario "El Pais" 25 marzo 2001. Francisco Peregil. Pag. 2. Especial Domingo. 
(4) Plan Nacional sobre Drogas (2001). Observatorio Español sobre Drogas. Informe $n^{\circ} 4$. Madrid: Plan Nacional sobre Drogas.

(5) Plan Nacional sobre Drogas (2002). Observatorio Español sobre Drogas. Informe $n^{\circ} 5$. Madrid: Plan Nacional sobre Drogas.

(6) Calafat, A.; Juan, M.; Becoña, E.; Fernández, C.; Gil, E.; Palmer, A.; Sureda, P. Y Torres, M.A. (2000). Salir de marcha y consumo de drogas. Madrid: Plan Nacional sobre Drogas.

(7) Miller, N.S. (1991). Nosology of drug and Alcohol Addiction. En: N. S. Miller (ed.) Comprehensive handbook of Drug and Alcohol Addiction. Nueva York: Marcel Dekker, Inc.

(8) Revista Cañamo n 39 pag 79, 88. La Cañamería global SL.

(9) Publicidad Doctor Cogollo pag. 9 Cañamo 40. La Cañamería global SL.

(10) Toro,G. (2002). Extasis Hervales. Cáñamo, 57, 100.

(11) Becoña, E. (1999). Bases teóricas que sustentan los programas de prevención de drogas. Madrid: Plan Nacional sobre Drogas.
(12) Bobes, J. y Calafat, A. (2000). De la neurobiología a la psicosociología del uso-abuso del cannabis. Adicciones, 12, Supl. 2, 7-17.

(13) Plan Nacional sobre Drogas (2003). Observatorio Español sobre Drogas. Informe $n^{\circ} 6$. Madrid: Plan Nacional sobre Drogas.

(14) Calafat, A., Fernández, C., Becoña, E., Gil, E., Juan, M. y Torres, M.A. (2000). Consumo y consumidores de cannabis en la vida recreativa. Adicciones, 12, Supl. 2, 197230.

(15) Kandel, D.B. (2002). Stages and pathways of drug involvement. Examining the gateway hypothesis. Cambridge: Cambridge University Press.

(16) Groff, S. (1988). Psicología transpersonal. Barcelona: Kairos.

(17) Krupitsky, E.M. y Grinenko, A.Y. (1996). Terapia psichedelica con ketamina (KPT) dell'alcoholismo, eficacia clinica e mecanismi di base. Eleusis, 14, 18-29.

(18) Revista Yerba. Edición Española de la revista Americana High Times. Central Media Young. № 1, Febrero 2001.

(19) Revista Cáñamo, n57, Septiembre 2002, pág. 95. La Cañamería Global SL. 